\title{
Microwave temperature and pressure measurements with the Odin satellite: II. Retrieval method
}

\author{
M. Ridal, D.P. Murtagh, F. Merino, J.R. Pardo, and L. Pagani
}

\begin{abstract}
The millimetre receiver on the Swedish satellite Odin, will be used for detection of the $118.750 \mathrm{GHz}$ oxygen line. The temperature and pressure will be determined from the output of a three-channel filter bank measurement. One frequency bin is centred over the emission-line frequency while the other two cover parts of the line wing, where the opacity is less, providing a useful signal at lower altitudes. The bandwidth of each channel is $40 \mathrm{MHz}$. The signal in the frequency bin covering the line centre is modeled by a high-resolution model including the Zeeman effect, developed by the Observatoire de Paris-Meudon. The other two $40 \mathrm{MHz}$ bins are modeled using the much faster standard Odin forward model, developed at the Department of Meteorology at Stockholm University together with Chalmers University of Technology. The operational retrievals employ an iterative method that uses simulated signals from a reference atmosphere as a lookup table for the pressure. The temperature is then calculated from the equation of hydrostatic equilibrium, and a new lookup table computed. This process is repeated until a convergence criterion is reached. Simulations, including known error sources, show that the temperature can be retrieved with a root mean square (rms) around $3 \mathrm{~K}$, in the altitude range $\sim 25-90 \mathrm{~km}$ using the operational temperature retrieval method (the filter bank method). A sub-millimetre receiver on board Odin will also be used to observe the oxygen line at $487.249 \mathrm{GHz}$. Both this line and the $118.750 \mathrm{GHz}$ line can be observed in high resolution $(150 \mathrm{kHz})$ for detailed studies of the Zeeman splitting. Retrievals from the high-resolution measurements are expected to give a precision of $\pm 2 \mathrm{~K}$ rms at that resolution. However, this kind of observation will occupy an entire spectrometer and will not be made on a regular basis.
\end{abstract}

Received 1 May 1999. Accepted 15 January 2001. Published on the NRC Research Press Web site at http://cjp.nrc.ca/ on 18 April 2002.

M. Ridal, ${ }^{1,2}$ D.P. Murtagh, ${ }^{3}$ and F. Merino. Department of Meteorology, Svante Arrhenius v. 12, Stockholm University, SE-10691 Stockholm, Sweden.

J.R. Pardo. ${ }^{4}$ DEMIRM, UMR 8540, Observatoire de Paris-Meudon, 61 avenue de l'Observatoire, 75014 Paris, France; NASA-Goddard Institute for Space Studies, 2880 Broadway, New York, NY 10025, U.S.A.; and George W. Downs Laboratory of Physics, MS 320-47, California Institute of Technology, 1200 E California Blvd., Pasadena, CA 91125, U.S.A.

L. Pagani. DEMIRM, URA 336 du CNRS, Observatoire de Paris-Meudon, 61 avenue de l'Observatoire, 75014 Paris, France.

${ }^{1}$ Corresponding author (email: martin@smhi.se).

${ }^{2}$ Present Address: SMHI (Swedish Meteorological and Hydrological Institute), SE-601 76 Norrköping, Sweden.

${ }^{3}$ Present Address: Department of Radio and Space Science, Chalmers University of Technology, SE-41296 Göteborg, Sweden.

${ }^{4}$ Present Address: Consejo Superior de Investigaciones Cientificas, IEM, Serrano 121, E-28006, Madrid, Spain. 
PACS Nos.: 07.57Yb, 94.10Dy, 95.75Rs

Résumé : Le récepteur millimétrique du satellite Odin a pour mission de détecter la raie à $118,750 \mathrm{GHz}$ de l'oxygène. Une batterie de filtres à trois canaux permet de déterminer la température et la pression. Une des fenêtres en fréquence est centrée sur le milieu de la raie d'émission, alors que les deux autres couvrent les ailes où l'opacité est plus faible, fournissant un signal utile à basse altitude. La largeur de chaque canal est $40 \mathrm{MHz}$. Le signal dans le canal central est modélisé par un modèle à haute résolution développé à l'Observatoire de Paris-Meudon et tient compte de l'effet Zeeman. Les signaux des deux canaux latéraux sont modélisés par le modèle standard Odin qui est beaucoup plus rapide de calcul et qui a été développé par le département de Météorologie de l'Université de Stockholm avec l'Université technique de Chalmers. Les résultats sont recouvrés par une méthode itérative qui utilise le signal simulé d'une atmosphère de référence comme table de recherche pour déterminer la pression. On calcule alors la température à l'aide de l'équation d'équilibre hydrostatique et une nouvelle table est générée. On répète le processus jusqu'à convergence. Des simulations, incluant les sources connues d'erreur montrent que la température peut être déterminée avec une valeur rms de $3 \mathrm{~K}$, aux altitudes variant de 25 à $90 \mathrm{~km}$ avec la procedure normale d'observation. Un détecteur submillimétrique à bord d'Odin a comme mission d'observer la raie de l'oxygène à 487,249 GHz. Ces deux raies peuvent être observées à haute résolution $(150 \mathrm{kHz})$ pour une étude détaillée de la séparation Zeeman. Le traitement de l'ensemble de ces mesures à haute résolution devrait mener à une précision de $\pm 2 \mathrm{~K}$ rms. Cependant, cela requiert l'utilisation en continu d'un spectromètre et ne sera donc pas fait sur une base continue.

[Traduit par la Rédaction]

\section{Introduction}

Odin is the third in a series of small Swedish satellites. Odin has been built in collaboration with Canada, Finland, and France, and will measure a number of atmospheric constituents for studies of middle-atmospheric chemistry and the dynamics of the stratosphere and mesosphere. The spacecraft is equipped with two instruments, a millimetre-sub-millimetre receiver (SMR) for both atmospheric and astronomical measurements and an optical instrument (OSIRIS) that is only used for aeronomy measurements [1].

Measurements of temperature and pressure using the $118.75 \mathrm{GHz} \mathrm{O}_{2}$ line will be performed by the SMR instrument. The routine measurements of the thermal emissions from this oxygen line will be made in three fixed-frequency filters (filter bank), one covering the line centre and two further out in the line wings. There is also the possibility of using a high-resolution spectrometer for measurements of this and the $487.25 \mathrm{GHz}$ oxygen line. The two different measurement modes are described in detail in Pardo et al. [2].

A complicating feature of the atmospheric oxygen lines is the Zeeman-splitting effect. The permanent magnetic dipole of the $\mathrm{O}_{2}$ molecule interacts with the Earth's geomagnetic field and causes a splitting of the rotational energy levels. Below $60 \mathrm{~km}$ the Zeeman splitting is negligible compared to the pressure broadening of the line. Further, the high rate of collisions effectively prevents the magnetic dipoles from orientating along the direction of the magnetic field. Above $\sim 60 \mathrm{~km}$ however, this effect becomes significant and needs to be taken into account when simulating $\mathrm{O}_{2}$ spectra $[2,3]$.

This work describes a retrieval method specifically designed for the filter bank measurements with the SMR instrument. The method, developed at the Department of Meteorology at Stockholm University, is similar to the method used to retrieve temperature and pressure from LIMS data [4].

In this paper, we first outline the physical requirements (Sect. 2) needed for the retrieval of temperature and pressure. The forward models used for simulating the satellite signals and for use in the retrieval models are fully described in ref. 2 but for completeness, briefly described in Sect. 3. Section 4 then follows with a short description of the different $\mathrm{O}_{2}$ measurement modes. In Sect. 5, we describe the retrieval methods, the operational in detail and the high-resolution retrieval briefly. A few examples 
of retrieved profiles follow in Sect. 6. We will also, in the last section, estimate the size of the main error sources and the effect they will have on the retrievals.

\section{Physical precepts}

Under the assumption that local thermodynamic equilibrium (LTE) applies, the radiation, $I$, coming from an atmospheric constituent is dependent on the amount of gas, the properties of the molecule, and the local temperature [5] according to

$I=B(T) N \varepsilon \mathrm{d} l$

where $B(T)$ is the blackbody function for temperature $(T), N$ is the number density of the emitting gas and $\varepsilon$ includes the properties of the molecule and of the absorption line in use, $\mathrm{d} l$ is the path length through the gas in which the measurement took place. The product $N \varepsilon$ is commonly known as the absorption coefficient.

The retrieval of the unknown mixing ratios of different atmospheric molecules thus requires a priori knowledge of the temperature. This required information can come from either climatological temperature and pressure profiles or from local simultaneous measurements. The latter are preferred since they are expected to give more accurate information than the climatology. The temperature itself can be derived from measurements of atmospheric species with well-known mixing ratios. Molecules with known mixing ratios are for example $\mathrm{O}_{2}$ and $\mathrm{CO}_{2}$. These have been used frequently in temperature measurements from satellites to determine the atmospheric temperature and pressure, see, for example, refs. 6-8.

A further assumption that has to be made is that the atmosphere is in hydrostatic equilibrium. Otherwise one cannot relate the pressure and temperature to each other.

Two advantages with using the $118.75 \mathrm{GHz}$ oxygen line is that the wavelength is long enough for the scattered solar radiation to be negligible and there are no "disturbing lines" that will affect the measured signal significantly.

\subsection{Zeeman splitting}

$\mathrm{O}_{2}$ has a permanent magnetic dipole that interacts with external magnetic fields, which results in a splitting of the rotational energy levels. This effect appears naturally in the atmosphere due to the geomagnetic field. Below $\sim 60 \mathrm{~km}$ it becomes insignificant because the frequency splitting is negligible compared with the total width of the oxygen lines and the high rate of collisions below that altitude range prevents the magnetic dipoles from effectively orientating along the magnetic-field direction. However, at altitudes above $\sim 60 \mathrm{~km}$ the effect has been shown to become important enough that it needs to be accounted for [3]. The effect for the $118.75 \mathrm{GHz} \mathrm{O}_{2}$ emission line can be seen in the right panel of figure 3 in ref. 2. The panel shows how the signal increases with altitude compared with a simulation with the magnetic field turned off. Below $70 \mathrm{~km}$ all the profiles approach zero indicating that the Zeeman effect becomes less important at lower altitudes. The figure clearly shows the importance of including the Zeeman effect above $60 \mathrm{~km}$.

There are several measurements confirming the importance of the Zeeman splitting in the upper stratosphere and lower mesosphere, see, for example, refs. 3, 9, or 10.

\subsection{Requirements}

The sensitivity of the constituent retrievals to an error in the temperature estimate have been investigated by Baron et al. [11]. They found that a root-mean-square (rms) error in the temperature of $3 \mathrm{~K}$ below $30 \mathrm{~km}$ and $5 \mathrm{~K}$ above, resulted in an error of less than 10\%, compared with the thermal noise that gave an error of $20-50 \%$ in the retrieved $\mathrm{ClO}$ profile. For ozone, the contribution from the temperature error source was less than 5\%, while the thermal noise gave an error of $10-30 \%$. 
The goal is thus to retrieve the temperature within $5 \mathrm{~K}$. We need a precision of this order to keep the error of the constituent retrievals small compared with the one due to thermal noise.

\section{The forward models}

There are two models that will be used in the retrieval and simulation of the $\mathrm{O}_{2}$ spectra. The first forward model was developed jointly by the Department of Meteorology at Stockholm University (MISU) and the Department of Radio and Space Science at Chalmers University of Technology (CTH) in Göteborg. It is described in detail by Eriksson et al. [12].

This model calculates absorption coefficients for a number of atmospheric layers and integrates the radiative-transfer equation along the observation path, taking refraction into account. The absorption coefficients are computed by a line-by-line calculation where the spectroscopic parameters are taken from the Jet Propulsion Laboratory (JPL) catalogue [13] and the HITRAN database [14]. The millimetrewave propagation model MPM93 [15] is used to model the complex refractive index of $\mathrm{O}_{2}, \mathrm{~N}_{2}$, and $\mathrm{H}_{2} \mathrm{O}$.

The model does not take the Zeeman-splitting effect into account. This effect gives, as already mentioned, a significant effect on the measured spectra and is therefore important to model correctly. For modeling the line centre, including the Zeeman effect, we instead use a radiative-transfer model developed at the Observatoire de Paris-Meudon (OPM). The model is described in more detail in ref. 2. This model includes, for the lower part of the atmosphere (up to $\sim 60 \mathrm{~km}$ ), different sets of spectroscopic parameters for molecular oxygen and water vapour taken from the works of Liebe [15-17] and Pardo et al. [2] with transition probabilities calculated directly from the Hamiltonian by Cernicharo [18] and line widths provided by Rosenkranz [19].

\section{Odin $\mathrm{O}_{2}$ measurements}

The sub-millimetre instrument on board Odin will be able to detect two $\mathrm{O}_{2}$ lines, one at $118.750 \mathrm{GHz}$ and the other at $487.249 \mathrm{GHz}$. The first line will be used for the operational temperature and pressure measurements while the latter is more suitable for high-resolution studies. A brief description of the two measurement modes will be given here but the details of these two modes are described in ref. 2 .

\subsection{Operational mode}

The measurements of the $118.750 \mathrm{GHz} \mathrm{O}_{2}$ line are made in three frequency bins each one is $40 \mathrm{MHz}$. The positions of these frequency bins are selected, primarily to cover as large an altitude interval as possible, but also to avoid other disturbing lines (e.g., ozone) that might affect the $\mathrm{O}_{2}$ spectra. The first channel covers the line centre $(118.730-118.770 \mathrm{GHz})$ and will give a useful signal in the altitude range 55 to $85 \mathrm{~km}$. The upper limit, around $85 \mathrm{~km}$, is determined by the decreasing amount of $\mathrm{O}_{2}$ and the change in atmospheric properties that will decrease the signal-to-noise ratio too much. The lower limit is determined by the increasing opacity of the atmosphere in that channel. Saturation is reached around $55-60 \mathrm{~km}$. Since the Zeeman effect becomes significant above $60 \mathrm{~km}$, this channel is the only one that will be affected by it. The effect it causes is that the total intensity within the frequency bin increases due to the different opacity distribution of the split line with respect to the unsplit line. The second channel is located fairly close to the line centre $(118.800-118.840 \mathrm{GHz})$ and will cover the region from $55-60 \mathrm{~km}$ down to $\sim 35 \mathrm{~km}$. The latter channel gives a useful signal from 35 down to about $25-30 \mathrm{~km}$. The spectral range of this channel is $119.180-119.220 \mathrm{GHz}$, which is rather far out in the line wing. The altitude intervals that each channel will cover may vary slightly depending on the atmospheric conditions. There are no overlapping emission lines from other compounds in these three bins except for the isotopic $\mathrm{O}_{2}$ line $\left({ }^{16} \mathrm{O}^{18} \mathrm{O}\right.$ at $\left.118.760 \mathrm{GHz}\right)$ that lies within the centre channel (see figure 3 in ref. 2). This line is however taken into account in the model calculations [2]. 


\subsection{High-resolution mode}

The SMR autocorrelator spectrometers (or backends) can provide a spectral resolution of $150 \mathrm{kHz}$. Consequently, they allow resolution of the Zeeman structure of the two $\mathrm{O}_{2}$ lines accessible to Odin receivers $(118.750$ and $487.249 \mathrm{GHz})$. This kind of measurement requires the use of an entire backend while the backends are mainly intended to obtain high-resolution minor constituent spectra. For that reason these observations will not be carried out in an operational way but only occasionally. They are important to carefully validate the radiative-transfer model in the mesosphere and will also provide information about the strength of the geomagnetic field.

\section{Temperature and pressure retrievals}

The operational retrieval model has been developed by MISU specifically for the three-channel approach. The method employed is an iterative lookup table method, which will be described below. The use of the optimal estimation method [20] is currently under investigation and preliminary results show that the retrievals are as good or even somewhat better than those obtained here. Which method to use will be decided during the validation of the data. The high-resolution retrievals, by the OPM, uses software, initially developed at the French-German-Spanish Institute of Millimetre Radio Astronomy (IRAM) and Observatoire de Grenoble, that allows fitting of the magnetic strength field and of the polarization angle of the receiver along with the temperature and pressure.

\subsection{The operational retrieval method}

The retrieval of temperature is based upon the assumption that the signal in a given filter is proportional to the $\mathrm{O}_{2}$ concentration and the local temperature, and hence the pressure. The MISU filter bank retrieval is an iterative method based on lookup tables.

As a first step, a reference atmosphere is used to compute a table of radiances as functions of pressure for each filter channel. Using the measured signals, the pressure for each tangent altitude are obtained from the table. It should be remembered that each channel covers a particular pressure range.

Combining the preliminary pressure information with pointing data from the attitude control system of the satellite, the temperature can be derived as a function of altitude (and pressure), through the equation of hydrostatic equilibrium

$T=-\frac{M g}{R \frac{\partial \ln (p)}{\partial z}}$

where $M$ is the mean molar mass for air, $g$ the acceleration due to gravity, $R$ is the ideal-gas constant, and $p$ represents the pressure distribution. Both $M$ (taken from the U.S. standard atmosphere) and $g$ are allowed to vary with altitude.

This yields the temperature in the altitude range of the retrieval, approximately $25-90 \mathrm{~km}$. The exact interval will vary with latitude and season, depending on the structure of the atmosphere. Below the retrieval interval, temperature soundings from the meteorological centres ECMWF or NCEP (National Centers for Environmental Prediction) analyses will be smoothly merged with the retrieved profile. Above the retrieval interval, a climatological profile or temperature measurements from OSIRIS, the optical instrument on board Odin [21], will be appended.

To ensure that the new atmosphere is in hydrostatic equilibrium, the merged temperature profile is integrated, using the integral form of (2), to obtain the pressure at all altitudes. A reference level in the retrieved interval is required as an initial condition in the calculation. The data at this level will be taken from ECMWF (European centre for medium-range weather forecasts) analyses.

The new atmosphere is used to compute a revised lookup table and the procedure is repeated until a convergence criterion is reached. The procedure is illustrated in Fig. 1. 
Fig. 1. The retrieval cycle.

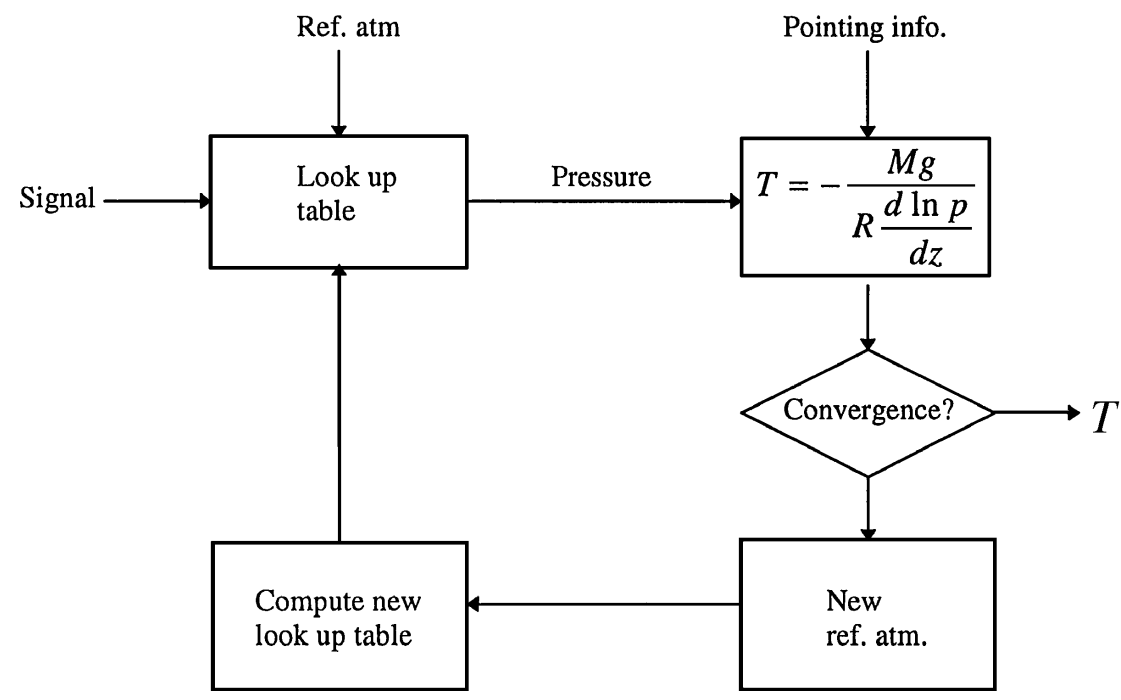

In evaluating (2), we must reduce the sensitivity of the derivative $\partial \ln (p) / \partial z$ to noise in either the signal or the pointing. The retrieved pressure is therefore smoothed using a running average with Gaussian weights. The standard deviation of the Gaussian was choosen to be $3.5 \mathrm{~km}$ as a compromise between stability and vertical resolution.

\subsection{High-resolution retrieval method}

The high-resolution retrievals are fitting routines specifically developed to treat the $\mathrm{O}_{2}$ spectroscopic observations to be performed occasionally using the high-resolution backends. Those observations and their special retrievals are aimed at studying the strength of the magnetic field and getting correct values of the temperature and $\mathrm{O}_{2}$ mixing ratio above $\sim 65 \mathrm{~km}$. This is possible since the high-resolution observing mode (see Sect. 4.2) can resolve the Zeeman components of the $\mathrm{O}_{2}$ emission line.

The high-resolution retrieval software has been developed by the OPM for the French-GermanSpanish Institute of Millimetre Radio Astronomy. Among other applications it allows us to fit, in the case of interest here, the magnetic-field strength and the angle of the polarization of the receiver along with the temperature, $\mathrm{O}_{2}$ mixing ratio, and pressure. The retrieval is based upon a generalized nonlinear least-squares algorithm previously described and used to analyze different sets of atmospheric data in refs. $3,10,22,23$, and 24 (the last reference includes retrievals from $\mathrm{O}_{2}$ spectra obtained with a balloon-borne sub-millimetre instrument).

The method has been tested using sets of synthetic spectra around $118.75 \mathrm{GHz}$ to which Gaussian noise has been added. In Fig. 2, we present a case where Gaussian noise of $1 \mathrm{~K}$ has been added to $100 \mathrm{kHz}$ resolution synthetic spectra of $\mathrm{O}_{2}$ corresponding to east-west paths with tangent altitudes spaced $3 \mathrm{~km}$ above the equator and the Greenwich meridian. Figure 2 shows that the "true" temperature profile is well retrieved, within $\pm 2 \mathrm{~K}$, for the altitude range $50-110 \mathrm{~km}$. The initial $T / P$ profile given to the retrieval algorithm was quite different with respect to the true profile, used to generate the synthetic spectra (more than $10 \mathrm{~K}$ apart in the altitude range 70-110 km). If the Zeeman-splitting calculations are excluded in the retrieval procedure the resulting temperature profile differs from the true profile by $15 \mathrm{~K}$ or more above $65 \mathrm{~km}$ (although the results are still good below that altitude). Other error sources described below should be applied when using this software to analyze real Odin data. 
Fig. 2. Retrieved temperatures from a set of synthetic spectra corresponding to the high-resolution mode. The spectra on the right show the residual after their fit. Details on generating the synthetic spectra and the retrieval itself are given in Sect. 5.2.
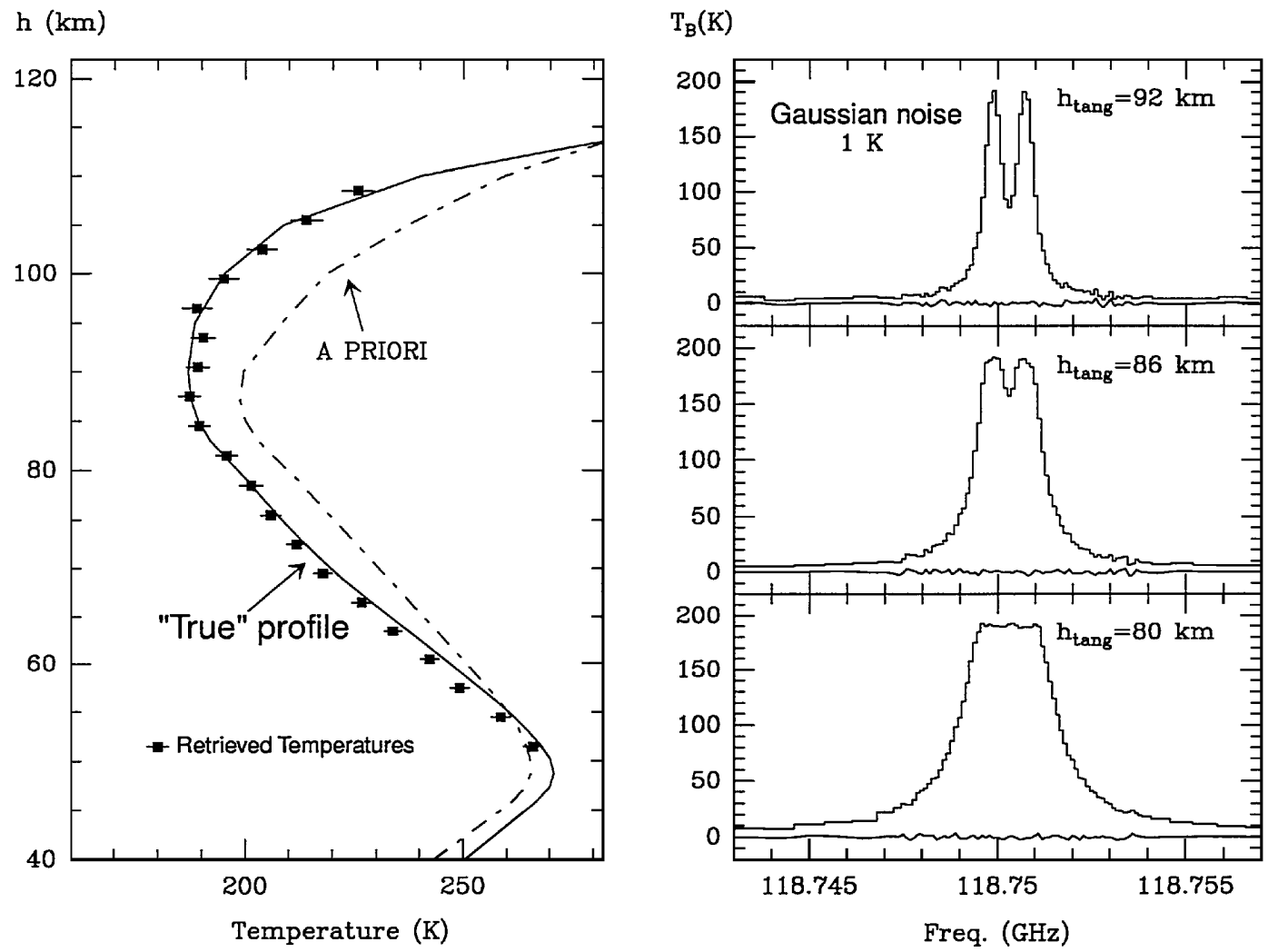

\section{Retrieved profiles from the operational mode}

A few examples of retrieved temperature profiles using synthetic spectra from the $118.75 \mathrm{GHz}$ line, using the filter bank method are presented here. The simulations include ozone and water vapour, which have lines that may disturb this oxygen line. Thermal noise is added to the simulated measurement and the starting profile used to calculate the first reference spectra is a standard temperature profile (indicated in the figures), unless otherwise stated. Vertical sampling of the simulation is $2 \mathrm{~km}$.

A retrieved profile for a standard atmosphere is shown in Fig. 3 where the left panel shows the retrieved (continuous line), the true profile (broken line), and the temperature profile used as starting profile (dotted-broken line). The right panel shows the difference between the retrieved and the true profile. This is a rather smooth profile and the retrieval model reproduces the true profile very well. The maximum error is $\sim 5 \mathrm{~K}(\mathrm{rms}=2.7 \mathrm{~K})$ within the retrieved interval, in this case, 28-96 km (indicated by dotted horizontal lines). Above and below this altitude range the true profile has been added instead of ECMWF analyses and climatology.

The number of iterations is, of course, determined by how different the initial reference profile and the true profile are. The larger the difference the more iterations. A very noisy profile will also require a few more iterations than a smooth "clean" profile. The number of iterations is also determined by the convergence criteria. A lower convergence limit requires more iterations but gives a better result than a higher limit. The higher limit on the other hand, requires fewer iterations, shorter computation time but a retrieved temperature profile that differs more from the true profile. 
Fig. 3. Left panel: Retrieved standard-temperature profile (continuous line), the true profile (broken line), and the starting profile (dotted-broken line). The dotted lines indicate retrieved altitude interval. Right panel: The ratio between the retrieved and the true profile.
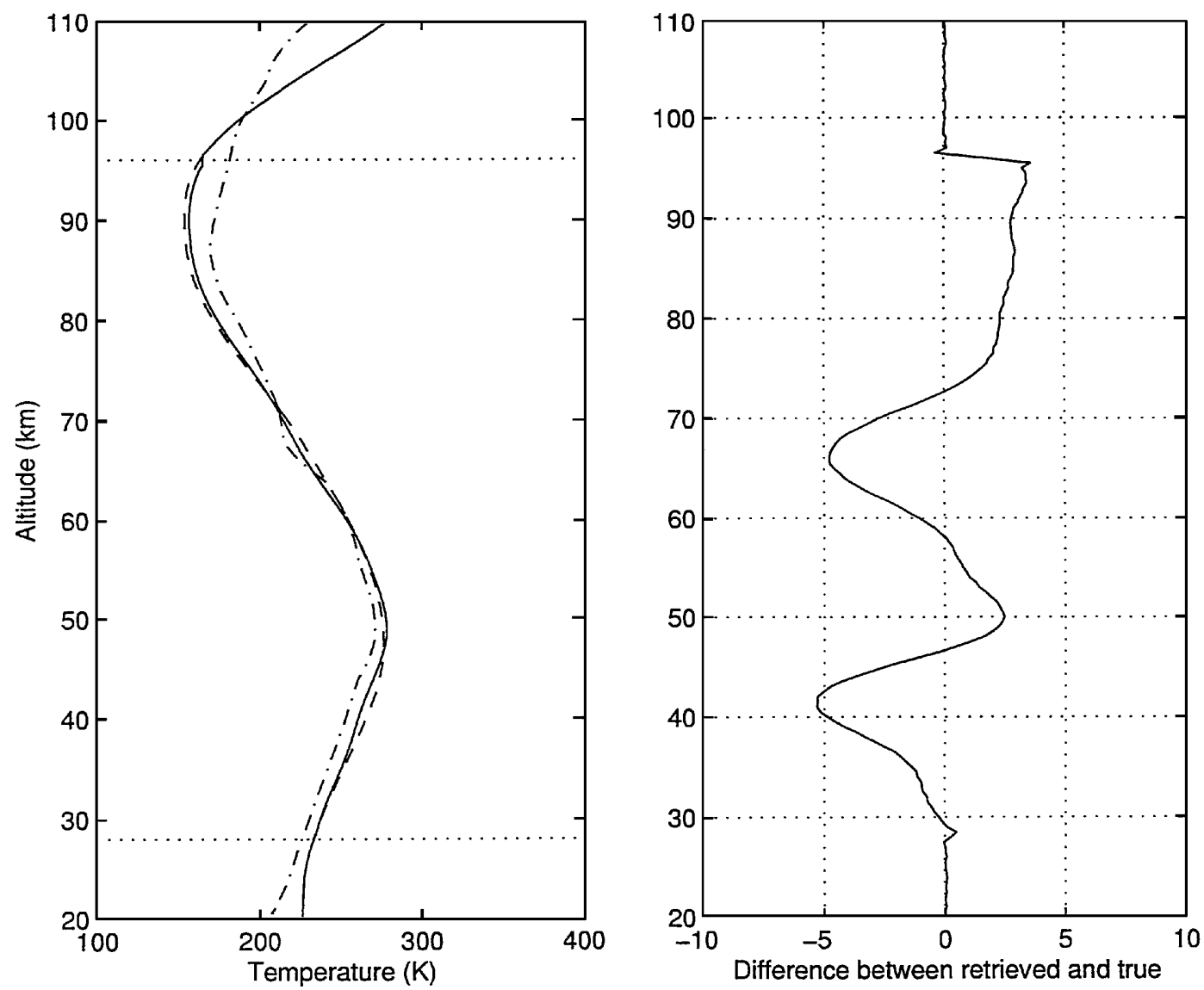

The accuracy of the pressure we obtain from the lookup table is, after the first iteration, within $3 \%$ rms up to about $80 \mathrm{~km}$. It improves on iteration and the final result is within $1 \% \mathrm{rms}$.

The smoothing procedure causes the underestimation of the temperature seen in Fig. 3 at the stratopause (around $50 \mathrm{~km}$ ). Above $70 \mathrm{~km}$, the true profile is overestimated. This is due to the curvature of the profile. Such overestimation or underestimation can be expected at the turning points of the temperature profile.

Due to the current method of smoothing the pressure profile, the model will not be able to reproduce the very small-scale structures of the temperature profile very well. The beam width and the antenna pattern will also affect the vertical resolution, but not as much as the pressure smoothing. The beam width at $118.75 \mathrm{GHz}$ is expected to be about $5 \mathrm{~km}$ for Odin.

To asses how large the vertical resolution of the retrieval model is we have calculated averaging kernels according to

$A=\frac{\partial x_{\text {ret }}}{\partial x_{\text {true }}}$

by perturbing the "true profile", $x_{\text {true }}$, layer by layer to see the effect on the retrieved profile, $x_{\text {ret }}$. These calculations showed that the vertical resolution is in the order of $10 \mathrm{~km}$ (see Fig. 4). Any structures with a vertical extent smaller than that cannot be resolved by the model. Future work will investigate better 
Fig. 4. Examples of averaging kernels at 35 (continuous line), 50 (broken line), and $70 \mathrm{~km}$ (dotted line).

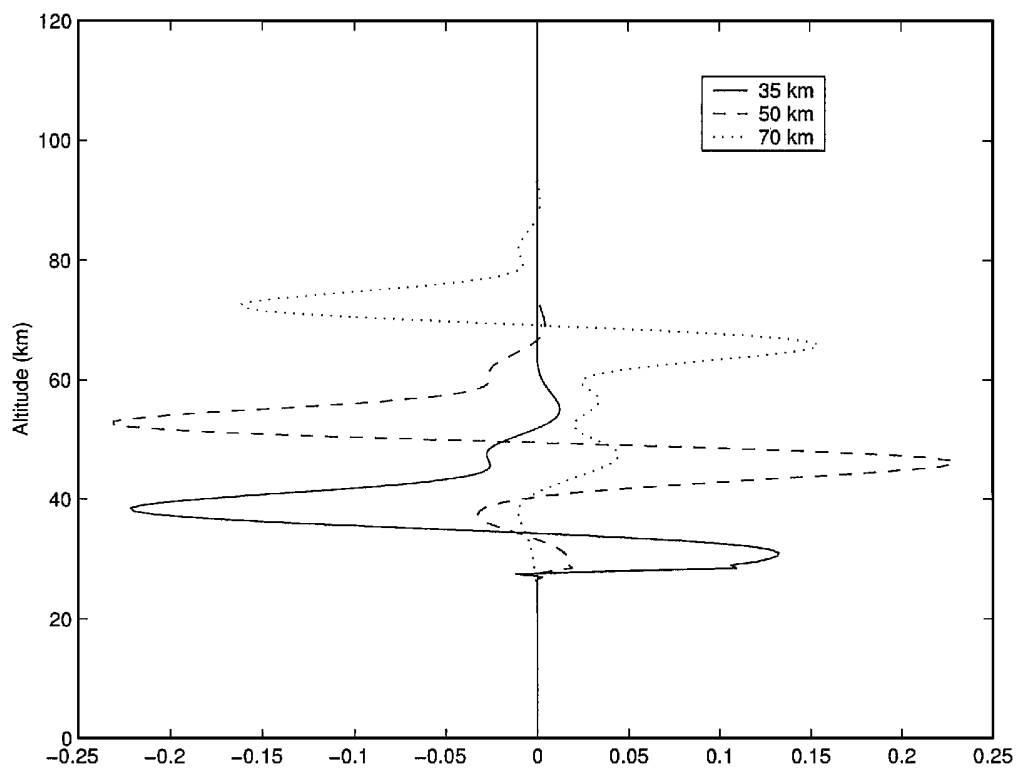

smoothing techniques and whether they help resolve the small-scale structures of the true temperature profile or not. On the other hand, the beam width will prevent any better vertical resolution than $5 \mathrm{~km}$.

One advantage of the smoothing is that the influence of thermal noise is reduced. When thermal noise is included, one or two extra iterations are required to reach convergence. The final result is however very much the same, which will be shown in the next section.

The simulations above have been made without considering Zeeman splitting. Its inclusion, apart from being necessary to obtain correct temperatures above $60 \mathrm{~km}$ also has the effect of raising the upper limit of the retrieval interval. The reason for this is the decreased opacity in the centre bin (see Sect. 4.1). During more detailed simulations the signal from the frequency bin in the line centre will be taken from the Zeeman model.

In our simulations, the "first guess" is always the same and may differ substantially from the true profile. During the operational retrievals the initial profile (first guess) will be taken from a climatological database to be as close as possible to the profile we expect to measure. This will improve the retrievals and fewer iterations will be needed. In the retrievals of the temperature from LIMS data, for example, the retrieved temperature was used as a starting point for the next retrieval [6].

\section{Error sources}

There are a number of error sources that can affect the retrieval. Here we attempt to estimate the effect that the most significant errors will have on the operational retrieval. These errors are expected to be the thermal noise, uncertainties in the pointing, calibration errors, and uncertainties in the forward models.

\subsection{Thermal noise}

The thermal noise of the receiver is estimated according to

$\sigma=\frac{T_{\text {sys }}}{\sqrt{ } \tau b}$ 
Fig. 5. Left panel: Ten retrieved temperature profiles (continuous lines) and the true profile (broken lines). All with thermal noise added differently (at random). Right panel: The ratio between the retrieved profiles and the true temperature profile.
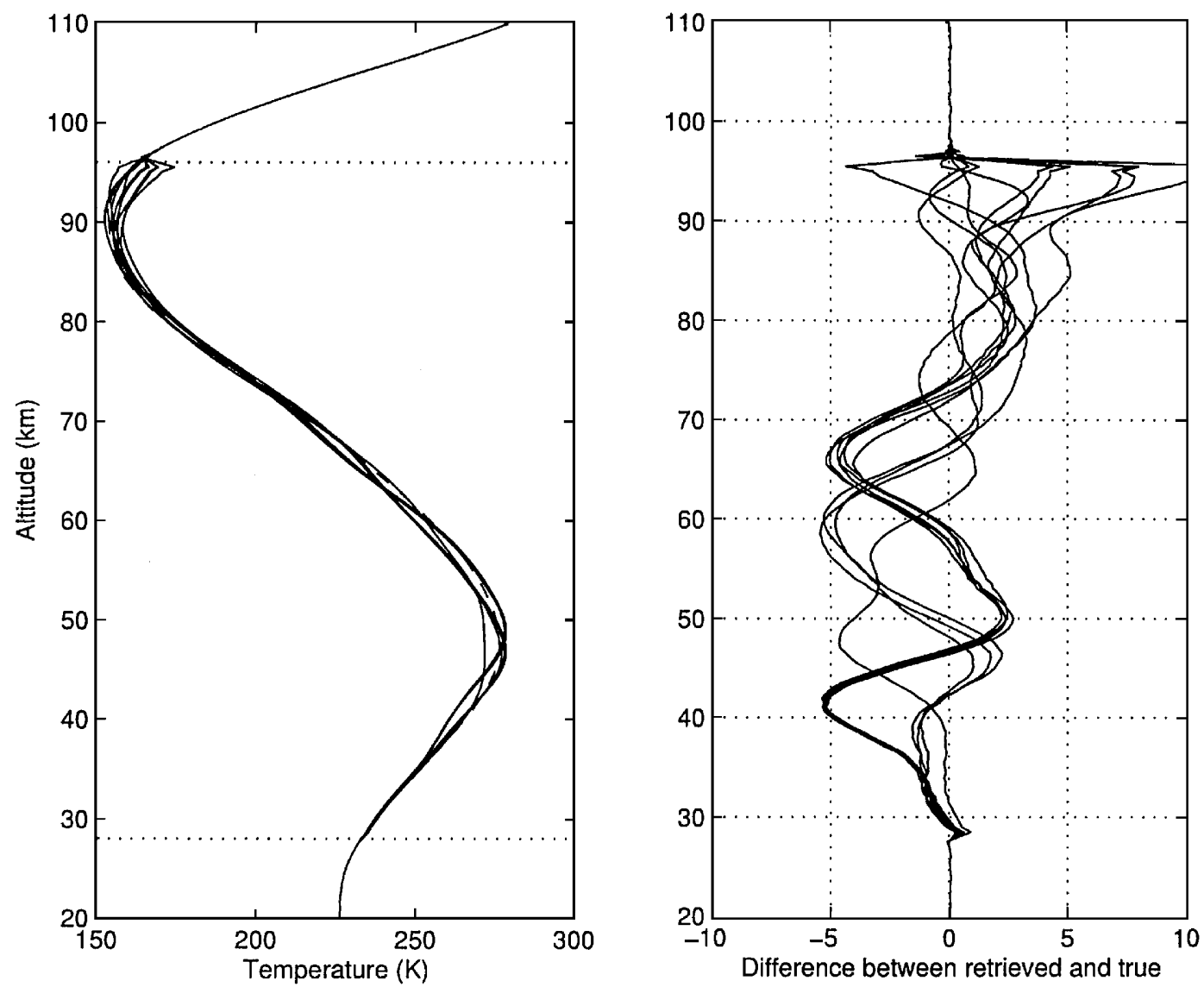

where $T_{\text {sys }}$ is the system temperature $(-600 \mathrm{~K}), \tau$ is the integration time (1-2 s), and $b$ is the bandwidth, which is $40 \mathrm{MHz}$ for the filter bank method. The thermal noise will thus be $0.07-0.1 \mathrm{~K}$. Simulations show that this level of thermal noise does not influence the retrieval significantly. This is because the smoothing of the pressure profile dampens the effect of noise during the lookup procedure. The remaining effects of thermal noise are illustrated in Fig. 5. The left panel shows ten retrievals, where each profile is retrieved from different simulations of noisy data. Compared to the retrieved temperature in Fig. 3, where the retrieval was made without added noise, we see that the error does not increase. The right panel shows the difference between the retrieved temperature profiles and the true profile.

We see that all retrievals give resulting profiles within $5 \mathrm{~K}$ of the true profile, up to $\sim 90 \mathrm{~km}$ where the signal-to-noise ratio is the limiting factor. Even though the noise gives a random result, there is a tendency towards an underestimation of the true profile up to about $70 \mathrm{~km}$ and an overestimation above. This is again due to the smoothing and the curvature of the profile.

\subsection{Errors in the pointing}

Two possible pointing errors have been considered. In the first case, the satellite looks systematically too high or too low. Secondly, a small stochastic error in the pointing where the step length between observations varies in a random manner. The latter will have an effect similar to thermal noise, giving a 
slightly incorrect pressure from the lookup table. The effect is also efficiently damped by the smoothing procedure.

A small systematic deviation in the pointing, however, will cause a displacement of the retrieved temperature and pressure profile in altitude. If the deviation is large (more than $3 \mathrm{~km}$ ) it will be quite obvious when the profiles from ECMWF are added below $25 \mathrm{~km}$. A $3 \mathrm{~km}$ displacement at $25 \mathrm{~km}$, will give an error in the pressure of about $10 \mathrm{hPa}$. This is a much larger error than is expected from the retrieval, which is less than $1 \mathrm{hPa}(3 \%)$ at that altitude.

Odin is expected to have an accuracy in pointing of less than $2 \mathrm{~km}$. Thus, the probability that the pointing error is of the magnitude that will cause problems is rather small.

\subsection{Calibration errors}

Calibration of the instrument is performed between each atmospheric scan by looking at a hot load on board the satellite and a cold load (the cosmic background). Both of these are affected by use of internal mirrors so that the telescope is included in the beam path. This could lead to some extra calibration errors. However, during the astronomy observations careful calibration against known sources will be used to characterize the telescope.

If the hot-load temperature is either fluctuating or if it is systematically too high (or too low), while the temperature of cold space is constant, it will cause an error in the measured brightness temperature. The error will increase with increasing atmospheric temperature. Tests show, however, that this error is expected to be small, not more than $\sim 0.1 \mathrm{~K}$, i.e., $0.02-0.03 \%$ of the measured signal.

To simulate this effect, we reduced the simulated signal at each altitude by $1 \%$, i.e., much more than expected. Again we retrieved ten profiles, first without adding thermal noise to see if the calibration errors will cause a systematic error, and then with thermal noise included. In the first case, we found that the error in the retrieved temperature profile caused by a calibration error of $1 \%$ was very small. A systematic structure on the retrieved profile could however be detected. All ten retrieved profiles had the same structure. The disturbance was visible between $35-55 \mathrm{~km}$, the region where the temperature maximum lies (the stratopause). At this altitude the signal is strongest, and thus the absolute error largest. With thermal noise included in the simulation of the measurement, the result was very similar to the results in Sect. 7.1. This indicates that the effect of the calibration error is much smaller than that of the thermal noise. If instead of a reduction of the signal, the calibration error should cause a higher signal than expected, i.e., we add $1 \%$ to the simulated signal, the result is similar.

\subsection{Errors in the forward model}

There are a number of things that may cause errors in the forward-model calculations. A number of these, such as the angular offset between different radiometers or the antenna response are well known for Odin. The astronomy measurements require more detailed specifications of these parameters than the aeronomy measurements. The spectral parameters, such as the pressure-broadening parameters and pressure shift, are hardly a problem since we use very broad filters (40 MHz). However, the spectroscopic-line databases, HITRAN and JPL, will introduce uncertainties that might be significant.

The MISU/CTH model is based on previous model work and compares very well to a similar, independently developed model by the French Odin group [12]. The two models used in this work, the MISU/CTH model and the OPM model, are also in good agreement.

\section{Conclusions}

By measuring the thermal emission of molecular oxygen in the atmosphere, the Odin satellite will be able to derive the temperature profile of the atmosphere in two ways. The first is the operational filter bank method, which will use thermal emissions from the $\mathrm{O}_{2}$ line at $118.75 \mathrm{GHz}$. The signal will be measured in three $40 \mathrm{MHz}$ wide frequency bins and the pressure and temperature profiles will be 
retrieved through an iterative process. The second is a high-resolution method with the possibility to observe both the $118.75 \mathrm{GHz}$ line and the oxygen line at $487.25 \mathrm{GHz}$. The high-resolution spectra will be used to get new accurate measurements of the Zeeman-splitting effect. Temperature and pressure can also be derived from these high-resolution spectra with the retrieval software developed at OPM. The temperature and pressure from the high-resolution measurements will be used to validate the operational retrievals.

Our simulations show that the operational retrieval method is expected to give good results. A temperature profile can be retrieved to a precision that lies within a root mean square of $3 \mathrm{~K}$, when the largest error sources are included. This will meet the requirements from the retrievals of other atmospheric constituents which use the temperature as input.

The vertical resolution of the retrievals is expected to be around $10 \mathrm{~km}$. The primary reason why this is not better is the smoothing of the pressure profile obtained from the lookup table. We currently use a Gaussian smoothing but are investigating better methods. Another limiting factor is the antenna beam width. Even though the beam width for Odin is about $5 \mathrm{~km}$ most of the information will come from the main lobe since the side lobes are very suppressed.

A number of different error sources were tested and shown to be of minor importance unless they assume unreasonably large values. The two errors that will have any effect on the retrieved profiles are the thermal noise and errors from the spectroscopic databases.

\section{Acknowledgements}

The work by the MISU group has been supported by grants from the Swedish National Space Board. J.R.P. gratefully acknowledges the financial support of the NASA-Goddard Institute for Space Studies, the Observatoire de Paris-Meudon, CNES, and Meteo-France for the development of this work.

\section{References}

1. D. Murtagh, U. Frisk, F. Merino et al. Can. J. Phys. 80 (2002). This issue.

2. J.R. Pardo, M. Ridal, D.P. Murtagh, and J. Cernicharo. Can. J. Phys. 80 (2002). This issue.

3. J.R. Pardo, L. Pagani, M. Gerin, and C. Prigent. J. Quantum Spectrosc. Radiat. Transfer, 54, 931 (1995).

4. J.C. Gille and F.B. House. J. Atmos. Sci. 28, 1427 (1971).

5. J.T. Houghton, F.W. Taylor, and C.D. Rodgers. Remote soundings of atmospheres. Cambridge University Press, Cambridge, New York. 1984.

6. J.C. Gille, J.M. Russel, P.L. Bailey, L.L. Gordely, E.E. Remsberg, J.H. Lienesch, W.G. Planet, F.B. House, L.V. Lyjak, and S.A. Beek. J. Geophys. Res. 89, 5147 (1984).

7. E.F. Fishbein, R.E. Cofield, L. Froidevaux et al. J. Geophys. Res. 101, 9983 (1996).

8. M.E. Hervig, J.M. Russel, L.L. Gordely et al. J. Geophys. Res. 101, 10277 (1996).

9. G.K. Hartmann, W. Degenhardt, M.L. Richards et al. Geophys. Res. Lett. 23, 2329 (1996).

10. B.J. Sandor and R.T. Clancy. Geophys. Res. Lett. 24, 1631. (1997).

11. P. Baron, P. Ricaud, J. de la Noë, J.E.P. Eriksson, F. Merino, M. Ridal, and D.P. Murtagh. Can J. Phys. 80 (2002). This issue.

12. J.E.P. Eriksson, F. Merino, D. Murtagh, P. Baron, P. Ricaud, and J. de la Noë. Can J. Phys. 80 (2002). This issue.

13. H.M. Poynter and D.E. Picket. Appl. Opt. 24, 2235 (1985).

14. L.S. Rothman, R.R. Gamache, R.H. Tipping et al. J. Quantum Spectrosc. Radiat. Transfer, 48, 469 (1992).

15. H.J. Liebe, G.A. Hufford, and M.G. Cotton. Proceedings NATO/AGARD Wave Propagation Panel. 52nd Meeting. 17-20 May 1993, Palma de Mallorca, Spain. 1993. No. 3, pp. 1-10.

16. H.J. Liebe. Radio Sci. 20, 1069 (1985).

17. H.J. Liebe. Int. J. Infrared Millimeter Waves, 10, 631 (1989).

18. J. Cernicharo. Thése de doctorat d'Etat, Université de Paris VI. 1988.

19. P.W. Rosenkranz. J. Quantum Spectrosc. Radiat. Transfer, 39, 287 (1988).

20. C.D. Rodgers. Rev. Geophys. 14, 609 (1976). 
21. E.J. Llewellyn, D.A. Degenstein, I.C. McDade et al. Applications of photonic technology-2. Edited by G.A. Lampropoulas and R.A. Lessard. Plenum Press, New York. 1997. pp. 627-632.

22. J.R. Pardo. Thése de doctorate, Université de Paris VI. 1996.

23. J.R. Pardo, E. Serabyn, and J. Cernicharo. J. Quantum Spectrosc. Radiat. Transfer, 68(4), 419 (2001).

24. J.R. Pardo, L. Pagani, G. Olofsson, P. Febvre, and J. Tauber. J. Quantum Spectrosc. Radiat. Transfer, 67(2), 169 (2000). 
